\title{
The Impact of Susceptible Human Immigrants on the Spread and Dynamics of Malaria Transmission
}

\author{
Alemu Geleta Wedajo ${ }^{1}$, Boka Kumsa Bole ${ }^{1}$, Purnachandra Rao Koya ${ }^{2}$ \\ ${ }^{1}$ Department of Mathematics, Wollega University, Nekemte, Ethiopia \\ ${ }^{2}$ Department of Mathematics, Hawassa University, Hawassa, Ethiopia
}

Email address:

alemugeleta@gmail.com (A. G. Wedajo), abitb2012@gmail.com (B. K. Bole), drkpraocecc@yahoo.co.in (P. R. Koya)

\section{To cite this article:}

Alemu Geleta Wedajo, Boka Kumsa Bole, Purnachandra Rao Koya. The Impact of Susceptible Human Immigrants on the Spread and Dynamics of Malaria Transmission. American Journal of Applied Mathematics. Vol. 6, No. 3, 2018, pp. 117-127.

doi: $10.11648 /$ j.ajam.20180603.13

Received: May 25, 2018; Accepted: June 26, 2018; Published: August 2, 2018

\begin{abstract}
Malaria is one of infectious diseases and has become the most public health issue especially in developing countries. Mathematically, the spread of malaria can be modeled to predict the dynamics of the outbreak of the disease. The present research studies the impact of migration of susceptible population on the dynamics of malaria transmission. In this paper an improved mathematical model is constructed based on a set of reasonable assumptions. Validity of the model is proved by verifying positivity of the solution. Mathematical analysis is carried out including equilibrium point analysis. Basic reproduction number of the model is determined so as to study the effect of migration parameter on the malaria outbreak. It has been observed that the migration parameter is directly proportional to the malaria outbreak. Hence, it is suggested that in order to keep the malaria outbreak under control, the migration parameter is required to be minimized. That is, migration of populations is recommended to reduce so as to reduce the impact of malaria outbreak.
\end{abstract}

Keywords: Malaria Outbreak, Reproduction Number, Migration Parameter, Numerical Simulation

\section{Introduction}

Malaria is an infectious disease caused by the Plasmodium parasite and transmitted among humans through bites of female Anopheles mosquitoes. It remains one of the most prevalent and lethal human infections throughout the world. An estimated $40 \%$ of the world's population lives in malaria endemic areas. Most malaria cases and deaths occur in subSaharan Africa [1].

It causes an estimated 300 to 500 million cases and 1.5 to 2.7 million deaths each year worldwide. Africa shares $80 \%$ of the cases and $90 \%$ of deaths [2]. The environmental conditions in the tropics are the prime factor for malaria being endemic. The moderate-to-warm temperatures, high humidity and water bodies allow mosquitoes and parasites to reproduce. The epidemiological patterns of malaria usually vary with season because of its dependence on transmission from mosquitoes. The infection can lead to serious complications affecting the brain, lungs, kidneys and other organs of human body. Clinical symptoms such as fever, body pains, chills and sweats may develop a few days after infected mosquito bites [3]. Malaria has also gained prominence in recent times since climate change or global warming is predicted. The incidence of malaria increases as the warming increases. Both the increases and fluctuations in temperature affect favorably the vector and parasite life cycles. This can cause reduced prevalence of the disease in cold areas, while it may increase in warm and hot areas. Thus, climate change can affect the malaria prevalence pattern by migration from lower latitudes to regions where the human population has not developed immunity to the disease.

Controlling of malaria is a challenging task due to many factors. The complexity of the disease control process, cost of the control program and resistance of the parasite to antimalarial drugs, and vectors to insecticides, are some of the challenges.

There is a variation in disease patterns and transmission dynamics from place to place, by season and according to varying environmental circumstances. The approaches in the 
planning and implementation of prevention and control activities also vary based on local realities.

Malaria cases are exacerbated by the high levels of HIV infection that weaken the immune system rendering people with HIV more susceptible to the disease [4]. It enhances mortality in advanced HIV patients by a factor of about $25 \%$ in non-stable malaria areas [5]. Since malaria increases morbidity and mortality, it continues to inflict major public health and socio-economic burdens in developing countries.

It is clear that poverty, while not a disease in itself, is a contributing factor not only for malaria but also for almost all diseases that face mankind. Because of poverty, communities may have poor sanitation and poor drainage, and these two factors allow the mosquitoes to breed in ever greater numbers. Poverty also means that people will not be able to afford the simple protection of a mosquito net or even screens for their windows.

A favorite hiding place for the Anopheles is in a dark moist room. With an increased number of vectors living with a human results in an increased chance of being bitten by an infected mosquito, which will in turn infect the human with the parasite [6].

Malaria has for many years been considered as a global issue. Many epidemiologists and other scientists have been investing their efforts in understanding the dynamics of malaria and to design the control strategies of its transmission. To complement the efforts of those scientists mathematicians have developed a significant and effective tool namely modeling technique of malaria. The models give insights into many aspects including the interaction between the human and mosquito populations, the dynamics of malaria, and also the controlling and eradication strategies of the transmission.

Mathematical modeling of malaria has been flourishing since the days of Ronald Ross who was awarded a Nobel Prize for his work in 1911. He developed a simple SIS model comprising of Susceptible, Infected, and Susceptible populations. It was based on the assumption that at any time the total population can be divided into two distinct human compartments. It was shown that malaria can be eliminated if the reproduction number is brought below a certain value. This value of reproduction number naturally depends on biological factors such as the biting rate and vectorial capacity [7].

For estimating infection and recovery rates a model is used assuming that population exposed to infection remains unchanged over time. This model has proved that reducing the number of mosquitoes is an inefficient control strategy and that had a little effect on the dynamics of malaria in areas of strong transmission [8].

The Ross-Macdonald mathematical model involves an interaction between infected human hosts and infected mosquito vectors. Bailey and Aron models take into account that acquired immunity to malaria depends on exposure i.e. the immunity is boosted by additional infections [9-11].

Tumwiine et al. used SIS and SI models in the human hosts and mosquito vectors respectively for the study of malaria epidemics that last for a short period in which birth and immunity to the disease were ignored. The system was in equilibrium only at the point of extinction that was neither stable nor unstable. However, some important results were revealed numerically [12].

Some recent papers have also included the effects of environment and that of the resistance to drugs [13-17]. Recently, some other researchers proposed ODE compartmental models to describe the spread of malaria [18, 19]. Compartment model for the spread of malaria with susceptible-infected-recovered-susceptible (SIRS) pattern for human and susceptible-infected (SI) pattern for mosquito populations have been developed [3, 20].

Yang, Wei, and Li proposed a model dividing respectively the human and vector populations into SIR and SI compartments [21].

The reproduction number $R_{0}$ is defined and shown that the disease-free equilibrium as well as endemic equilibrium exists and is stable $[3,20,21]$.

The immigration of human population is not taken in to consideration in the model [20]. Hence, this need not be a reasonable model due to the following reasons: the immigration of human population has a potential impact on the dynamics of malaria, controlling strategies and eventually eradication.

In the present study the existing model is improved by including migration of susceptible human population. The main objective of this study is to understand the impact of susceptible immigrations on the transmission dynamics and the spread of malaria disease.

\section{Model Assumptions}

i. Immigration in to the human susceptible compartment alone is considered.

ii. The number of infected people increases at a rate proportional to the number of possible contacts of infectious mosquito and susceptible human populations.

iii.Humans move from susceptible to infectious compartment with a progression rate of $\beta_{h}$.

iv. The transfer rate of humans from infectious to recovered compartment is proportional to the number of infectious humans. Humans move from infectious to recovered compartments with a progression rate of $\gamma_{h}$.

v. A human can die at any stage due to natural causes. Therefore $\alpha_{h}$ is taken as natural death rate in every human compartment.

vi. The recovered human individuals do not enter into the susceptible class again,

vii. Mosquitoes do not recover from malaria.

\section{Formulation of the Model}

The model of malaria transmission considered in this study is $S I R$ for human population and $S I$ for mosquito 
population. The model is formulated for the spread of malaria in both the human and mosquito populations with the total respective population sizes at time $t$ are denoted by $N_{h}(t)$ and $N_{v}(t)$. Human population is compartmentalized into epidemiological classes as susceptible $S_{h}(t)$, infected $I_{h}(t)$, and recovered $R_{h}(t)$ compartments. Similarly, mosquito population is compartmentalized as susceptible $S_{v}(t)$ and infected $I_{v}(t)$ vector populations. The vector component of the model does not include an immune class as mosquitoes never recover from the infection, that is, their infective period ends with their death due to their relatively short lifecycle. Thus, the immune class in the mosquito population is negligible and hence omitted. Also, the natural death occurs with equal rates in all mosquito compartments.

The model can be used for diseases that persist in a population for a long period of time with vital dynamics. The basic model incorporates a set of assumptions. Both the human and vector total population sizes are assumed to be constant. The recovered individuals in human population develop permanent immunity. The populations in compartments of both humans and vectors are non-negative, and so is the case with all the parameters involved in the model as it is evident from Table 1 .

Table 1. Notations and descriptions of state variables and parameters.

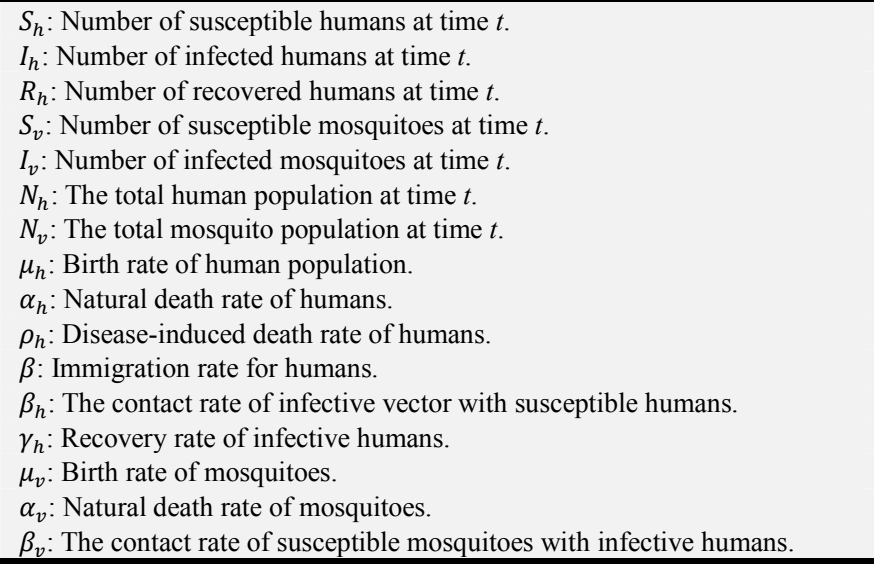

All newborns are susceptible to infection. The development of malaria starts when the infectious female mosquito bites the susceptible human. The vectors do not die from the infection or otherwise are harmed.

Individuals move from one class to the other as their status with respect to the disease.

Humans enter the susceptible class through birth rate $\mu_{h}$ and immigration rate $\beta$ and leave through death rate $\alpha_{h}$, and infective rate $\beta_{h} S_{h}$.

All human individuals, whatever may be the status, are subject to a natural death which occurs at a rate $\alpha_{h}$, and disease induced death rate $\rho_{h}$.

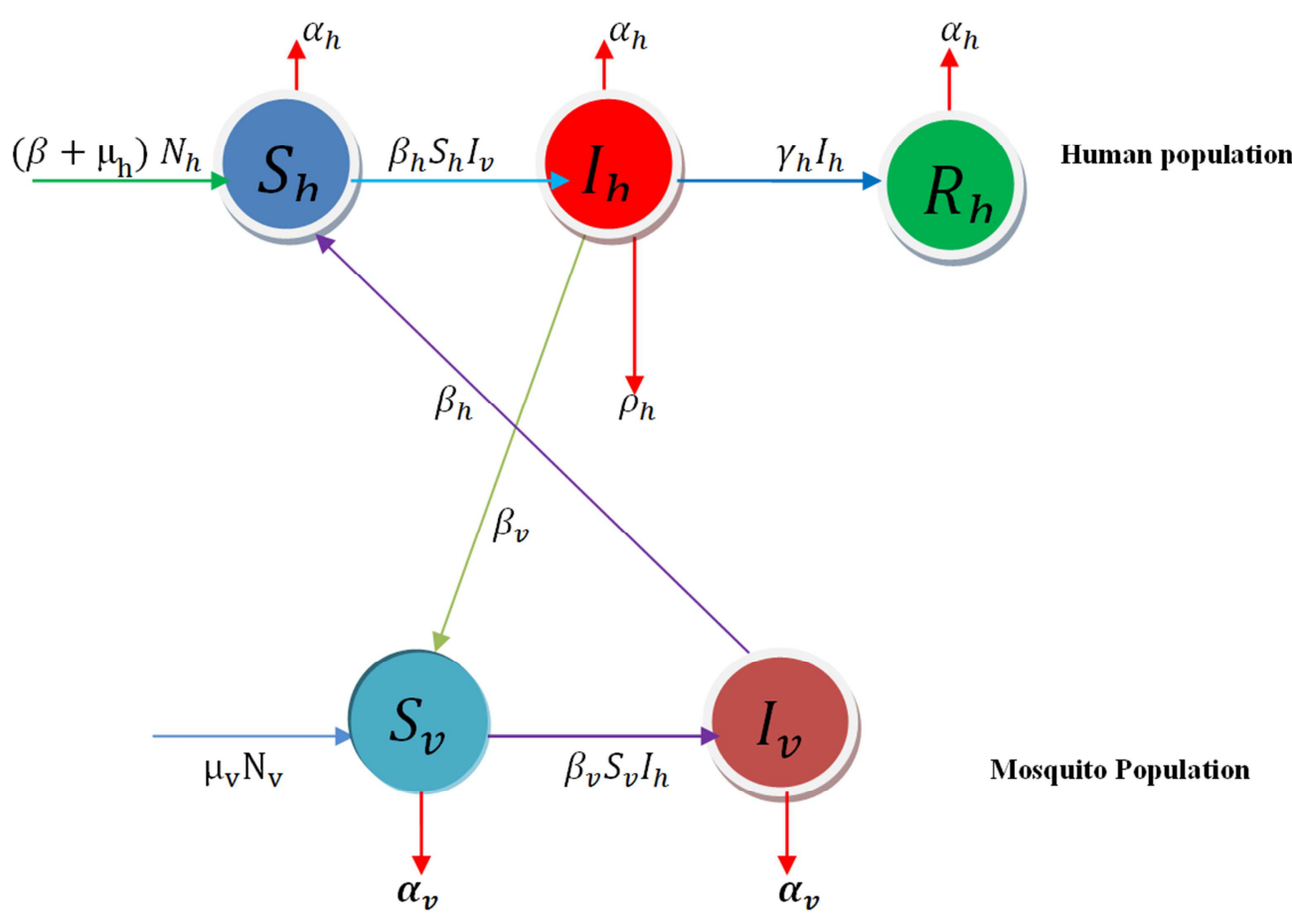

Figure 1. The flow diagram of malaria compartmental model.

With the inclusion of the model assumptions, notations of variables and parameters, the system of ordinary differential equations describing the dynamics of malaria in both the human and mosquito populations can be formulated as

$$
\begin{aligned}
& \frac{d S_{h}}{d t}=\left(\beta+\mu_{h}\right) N_{h}-\beta_{h} S_{h} I_{v}-\alpha_{h} S_{h} \\
& \frac{d I_{h}}{d t}=\beta_{h} S_{h} I_{v}-\rho_{h} I_{h}-\gamma_{h} I_{h}-\alpha_{h} I_{h}
\end{aligned}
$$




$$
\begin{gathered}
\frac{d R_{h}}{d t}=\gamma_{h} I_{h}-\alpha_{h} R_{h} \\
\frac{d S_{v}}{d t}=\mu_{v} N_{v}-\beta_{v} S_{v} I_{h}-\alpha_{v} S_{v} \\
\frac{d I_{v}}{d t}=\beta_{v} S_{v} I_{h}-\alpha_{v} I_{v}
\end{gathered}
$$

with initial conditions

$$
S_{h}(0)=S_{h 0}, I_{h}(0)=I_{h 0}, R_{h}(0)=R_{h 0},
$$

and

$$
\mathrm{S}_{\mathrm{v}}(0)=\mathrm{S}_{\mathrm{v} 0}, \mathrm{I}_{\mathrm{v}}(0)=\mathrm{I}_{\mathrm{v} 0}
$$

The total population sizes $N_{h}$ and $N_{v}$ can be determined by

$$
\begin{gathered}
S_{h}+I_{h}+R_{h}=N_{h} \\
S_{v}+I_{v}=N_{v}
\end{gathered}
$$

In this model, (i) The terms $\mu_{h} N_{h}$ and $\mu_{v} N_{v}$ denote the birthrates of human and mosquito populations respectively; (ii) $\beta N_{h}$ is the rate of immigration of susceptible humans (iii) The terms $\alpha_{h} S_{h}, \alpha_{h} I_{h}$ and $\alpha_{h} R_{h}$ refer to the removal rates of susceptible, infected and recovered humans due to natural deaths. (iv) the terms $\alpha_{v} S_{v}$ and $\alpha_{v} I_{v}$ are the rates of natural deaths of susceptible and infected mosquito populations (v) the term $\rho_{h} I_{h}$ is the number of removed humans because of the disease (vi) $\gamma_{h} I_{h}$ is the rate of recovered human population (vii) the term $\beta_{h} S_{h} I_{v}$ denotes the rate at which the susceptible human hosts $S_{h}$ get infected by the infective mosquito vector $I_{v}$ and (viii) $\beta_{v} S_{v} I_{h}$ refers to the rate at which the susceptible mosquitoes $\mathrm{S}_{\mathrm{v}}$ are infected by the infective human hosts $I_{h}$.

However, both the terms $\beta_{h} S_{h} I_{v}$ and $\beta_{v} S_{v} I_{h}$ are important parts of the model since they describe the interactions between the human and mosquito populations.

\section{Mathematical Analysis of the Model}

The mathematical analysis of the model described by the system (1) to (5) is presented here.

The rate of change of total human population size $N_{h}(t)=$ $S_{h}(t)+I_{h}(t)+R_{h}(t)$ can be obtained using equations (1) to (3) as

$$
\begin{array}{r}
\frac{d}{d t} N_{h}(t)=\frac{d}{d t} S_{h}(t)+\frac{d}{d t} I_{h}(t)+\frac{d}{d t} R_{h}(t) \\
=\left(\beta+\mu_{h}-\alpha_{h}\right) N_{h}-\rho_{h} I_{h}
\end{array}
$$

Similarly, the rate of change of total mosquito population size $N_{v}(t)=S_{v}(t)+I_{v}(t)$ can be obtained using equations (4) to (5) as

$$
\frac{d}{d t} N_{v}(t)=\frac{d}{d t} S_{v}(t)+\frac{d}{d t} I_{v}(t)=\mu_{v} N_{v}-\alpha_{v}\left(S_{v}+I_{v}\right)
$$

\subsection{Positivity of Solutions}

In order that the model equations (1) to (5) are epidemiologically meaningful and well posed it is appropriate to show that all the state variables are non- negative. This requirement is stated as a theorem and its proof is provided as follows:

Theorem 1: If $\mathrm{S}_{\mathrm{h}}(0)>0, \mathrm{I}_{\mathrm{h}}(0)>0, \mathrm{R}_{\mathrm{h}}(0), \mathrm{S}_{\mathrm{v}}(0)>0$ and $\mathrm{I}_{\mathrm{v}}(0)>0$, then the solution region $\left\{\mathrm{S}_{\mathrm{h}}(\mathrm{t}), \mathrm{I}_{\mathrm{h}}(\mathrm{t}), \mathrm{R}_{\mathrm{h}}(\mathrm{t}), \mathrm{S}_{\mathrm{v}}(\mathrm{t}), \mathrm{I}_{\mathrm{v}}(\mathrm{t})\right\}_{\text {of }}$ the system of equations (1) to (5)is always non negative.

Proof: To show the positivity of the solution of the dynamical system comprising the equations (1) to (5), each differential equation is considered and shown that its solution is positive.

Positivity of susceptible human population: Consider the differential equation (1) of the dynamical system $d S_{h} / d t=$ $\left(\beta+\mu_{h}\right) N_{h}-\beta_{h} S_{h} I_{v}-\alpha_{h} S_{h}$. Since $\left(\beta+\mu_{\mathrm{h}}\right) \mathrm{N}_{\mathrm{h}}$ is a positive quantity, the equation can be expressed as an inequality as $d S_{h} / d t \geq-\beta_{h} S_{h} I_{v}-\alpha_{h} S_{h}$. Using the technique of separation of variables and up on integration gives $S_{h}(t) \geq S_{h 0} e^{-\int_{0}^{t}\left(\beta_{h} I_{v}+\alpha_{h}\right) d s}$. But, for any value of the exponent, the exponential term is always a non-negative quantity, that is $e^{-\int_{0}^{t}\left(\beta_{h} I_{v}+\alpha_{h}\right) d s} \geq 0$. Also it is assumed that $S_{h 0}>0$. Thus, it is clear from the solution that $S_{h}(t)$ is positive.

Positivity of infective human population: Considering the differential equation (2) of the dynamical system $d I_{h} / d t=$ $\beta_{h} S_{h} I_{v}-\left(\rho_{h}+\gamma_{h}+\alpha_{h}\right) I_{h} \geq-\left(\rho_{h}+\gamma_{h}+\alpha_{h}\right) I_{h}$. The equation can be expressed as an inequality as $d I_{h} / d t \geq$ $-\left(\rho_{h}+\gamma_{h}+\alpha_{h}\right) I_{h}$. Using the technique of separation of variables and up on integration gives $I_{h}(t) \geq I_{h 0} e^{-\int_{0}^{t}\left(\rho_{h}+\gamma_{h}+\alpha_{h}\right) d s}>0$. But, for any value of the exponent, the exponential term is always a non-negative quantity, that is $e^{-\int_{0}^{t}\left(\rho_{h}+\gamma_{h}+\alpha_{h}\right) d s} \geq 0$. Also it is assumed that $\mathrm{I}_{\mathrm{h} 0}>0$. Thus, it is clear from the solution that $\mathrm{I}_{\mathrm{h}}(\mathrm{t})$ is positive.

Positivity of infective human population: From the differential equation (3) it can be expressed as $d R_{h} / d t=$ $\gamma_{h} I_{h}-\alpha_{h} R_{h} \geq-\alpha_{h} R_{h}$. The equation can be expressed as an inequality as $d R_{h} / d t \geq-\alpha_{h} R_{h}$. Using the technique of separation of variables and up on integration gives $R_{h}(t) \geq$ $R_{h 0} e^{-\int_{0}^{t} \alpha_{h} d s}>0$. But, for any value of the exponent, the exponential term is always a non-negative quantity, that is $e^{-\int_{0}^{t} \alpha_{h} d s} \geq 0$. Also it is assumed that $\mathrm{R}_{\mathrm{h} 0}>0$. Thus, it is clear from the solution that $\mathrm{R}_{\mathrm{h}}(\mathrm{t})$ is positive.

Positivity of susceptible mosquito population: Consider the differential equation (4) of the dynamical system $d S_{v} / d t=$ $\mu_{v} N_{v}-\beta_{v} S_{v} I_{h}-\alpha_{v} S_{v}$. Since $\mu_{\mathrm{v}} \mathrm{N}_{\mathrm{v}}$ is a positive quantity, the equation can be expressed as an inequality as $d S_{v} / d t \geq$ $-\beta_{v} S_{v} I_{h}-\alpha_{v} S_{v}$. Using the technique of separation of variables and up on integration gives $S_{v}(t) \geq S_{v 0} e^{-\int_{0}^{t}\left(\beta_{v} I_{h}+\alpha_{v}\right) d s}$. But, for any value of the exponent, the exponential term is always a non-negative quantity, that is $e^{-\int_{0}^{t}\left(\beta_{v} I_{h}+\alpha_{v}\right) d s} \geq 0$. Also it is assumed that $\mathrm{S}_{\mathrm{v} 0}>0$. Thus, it is clear from the solution that $\mathrm{S}_{\mathrm{v}}(\mathrm{t})$ is positive.

Positivity of infective mosquito population: Consider the differential equation (5) of the dynamical system $d I_{v} / d t=$ $\beta_{v} S_{v} I_{h}-\alpha_{v} I_{v}$. The equation can be expressed as an inequality as $d I_{v} / d t \geq-\alpha_{v} I_{v}$. Using the technique of 
separation of variables and up on integration gives $I_{v}(t) \geq$ $I_{v 0} e^{-\int_{0}^{t} \alpha_{v} d s}$. But, for any value of the exponent, the exponential term is always a non-negative quantity, that is $e^{-\int_{0}^{t} \alpha_{v} d s} \geq 0$. Also it is assumed that $\mathrm{I}_{\mathrm{v} 0}>0$. Thus, it is clear from the solution that $\mathrm{I}_{\mathrm{v}}(\mathrm{t})$ is positive.

The five dimensional solution space shows that all the solutions are positive. Hence, the feasible region containing all the solutions of the system of equations (1)-(5) is given by the set $\Omega=\left\{\left(S_{h}, I_{h}, R_{h}, S_{v}, I_{v}\right) \in \mathbb{R}^{+5}\right\}$. Here the quantities $S_{h}, I_{h}, R_{h}, S_{v}, I_{v}$ are all non - negatives.

\subsection{Scaling of the Model}

In order to simplify analysis of the malaria model given by the equations (1)-(5), it is recommended to work with fractional quantities instead of actual quantities. The fractional quantities can be achieved by scaling the model variables and parameters.

Let the scaled proportions for the quantities $S_{h}, I_{h}, R_{h}, S_{v}$ and $I_{v}$ be chosen as $S_{h}=N_{h} s_{h}, I_{h}=N_{h} i_{h}, R_{h}=N_{h} r_{h}, S_{v}=$ $N_{v} s_{v}, I_{v}=N_{v} i_{v}$. The original time coordinate $\left(\mu_{h} t\right)$ is transferred to the scaled time coordinate ast. Thus, the scaled version of the equations (1)-(5) then takes the form as

$$
\begin{aligned}
& \frac{d s_{h}}{d t}=1+\xi-\mathrm{B} s_{h} i_{v}-\alpha s_{h} \\
& \frac{d i_{h}}{d t}=\mathrm{B} s_{h} i_{v}-(\alpha+\gamma) i_{h} \\
& \varepsilon \frac{d i_{v}}{d t}=\vartheta\left(1-i_{v}\right) i_{h}-\delta i_{v}
\end{aligned}
$$

Similarly, the scaled initial conditions are $s_{h}(0)=$ $s_{h 0}, i_{h}(0)=i_{h 0}, i_{v}(0)=i_{v 0}$. Here in (10) to (12) the dimensionless parameters represent the following expressions:

$$
\begin{aligned}
\varepsilon=\left(\mu_{h} / \mu_{v}\right), \xi= & \frac{\beta}{\mu_{h}}, \mathrm{~B}=\frac{\beta_{h} N_{v}}{\mu_{h}}, \alpha=\frac{\alpha_{h}}{\mu_{h}}, \gamma=\frac{\rho_{h}+\gamma_{h}}{\mu_{h}}, \vartheta \\
& =\frac{\beta_{v} N_{h}}{\mu_{v}}, \delta=\frac{\alpha_{v}}{\mu_{v}}
\end{aligned}
$$

Clearly it is true that the scaling of the model reduces the population sizes of both human and mosquito to one unit. That is, $s_{h}+i_{h}+r_{h}=1$ and $s_{v}+i_{v}=1$. Equivalently, these can be expressed as $r_{h}=1-s_{h}-i_{h}$ and $s_{v}=1-i_{v}$

\subsection{Isease Free Equilibrium}

Disease - free equilibrium points are steady state solutions where there is no malaria in the human population or plasmodium parasite in the mosquito population.

The model compartments can be classified in to two categories: infectious and non-infectious categories. The human and mosquito infectious compartments are $i_{h}$ and $i_{v}$. The absence of the disease implies that $i_{h}=0$ and $i_{v}=0$.

The disease free equilibrium point requires the conditions $\frac{d s_{h}}{d t}=\frac{d i_{h}}{d t}=\frac{d i_{v}}{d t}=0$ and $i_{h}=0$ and $i_{v}=0$. The substitution of these requirements in the non -linear system (10) to (12) leads to the result $1+\xi-\alpha s_{h}=0$ giving $s_{h}=[[1+\xi] / \alpha]$.
Thus the disease - free equilibrium point of malaria model (2.10) to (2.12) is given by

$$
E_{0}=\left(s_{h}^{0}, i_{h}^{0}, i_{v}^{0}\right)=\left\{\frac{1+\xi}{\alpha}, 0,0\right\}
$$

In (13), $E_{0}$ represents the state in which there is no infection in the society i.e., either in human or mosquito population. That is, the malaria disease is absent.

\subsection{Basic Reproduction Number}

The reproduction number $\mathrm{R}_{0}$ is defined as the number of secondary infections that one infectious individual would create over the duration of the infectious period, provided that everyone else is susceptible. Here the next generation operator approach as described by Diekmann et al., (1990) is used to find an expression for the basic reproduction number. Reproduction number $R_{0}$ is the threshold for many epidemiology models. It determines whether a disease can invade in a population or not. If $R_{0}<1$ each infected individual produces on average less than one new infected individual so the disease is expected to die out. On the other hand if $R_{0}>1$, each individual produces more than one new infected individual so that the disease is expected to spread in the population. This means that the value of the threshold quantity $R_{0}$ for eradicating the disease is to be reduced to less than one.

The expression for the reproduction number $R_{0}$ is determined here using the next generation approach. The following steps are followed to compute $R_{0}$ :

The basic reproduction number cannot be determined from the structure of the mathematical model alone but from the definition of infected and uninfected compartments. Assuming that there are $n$ compartments in the model and of which the first $m$ compartments are of infected individuals [22].

Let the column vector $V_{i}(x)$ be defined as $V_{i}(x)=$ $V_{i}^{-}(x)-V_{i}^{+}(x)$. Here $V_{i}^{+}(x)$ is the rate of transfer of individuals into compartment $i$ by all means. Similarly, $V_{i}^{-}(x)$ is the rate of transfer of individuals out of compartment $i$. It is assumed that each function is continuously differentiable at least twice with respect to every dependent variable.

In general, a disease transmission model consists of nonnegative initial conditions together with a system of equations as follows

$$
\dot{x}_{i}=h_{i}(x)=F_{i}(x)-V_{i}(x), i=1,2,3, \ldots n
$$

Herex denotes the time derivative representing the rate of change of $x$.

The next step is the computation of the square matrices $F$ and $V$ of order $m \times m$. Here $m$ denotes the number of infected classes. Now, the matrices $F$ and $V$ are defined as $F=\left[\frac{\partial F_{i}}{\partial x_{j}}\left(x_{0}\right)\right]$ and $V=\left[\frac{\partial V_{i}}{\partial x_{j}}\left(x_{0}\right)\right]$ with $1 \leq i, j \leq m$. Here, $F$ is non-negative; $V$ is non-singular; and $x_{0}$ is the disease - free equilibrium point or DFE point.

Since $F$ is non-negative and $V$ non-singular, then $V^{-1}$ isnon-negative and also $F V^{-1}$ is nonnegative. Hence the matrix $F V^{-1}$ is called the next generation matrix for the 
model.

Finally the basic reproduction number $R_{0}$ is given by

$$
R_{0}=\rho\left(F V^{-1}\right)
$$

Here in (14), $\rho(A)$ denotes the spectral radius of matrix $A$ and the spectral radius is defined as the biggest non-negative eigenvalue of the matrix.

In case of the present model, $F_{i}$ and $V_{i}$ are constructed as

$$
\begin{gathered}
F_{i}=\left[\begin{array}{c}
\mathrm{B} s_{h} i_{v} \\
\vartheta i_{h}-\vartheta i_{h} i_{v}
\end{array}\right] \\
V_{i}=\left[\begin{array}{c}
(\alpha+\gamma) i_{h} \\
\delta i_{v}
\end{array}\right]
\end{gathered}
$$

The partial derivatives of (15) with respect to $\left(i_{h}, i_{v}\right)$ and the Jacobian matrix of $F_{i}$ at the disease - free equilibrium point (13) takes the form as

$$
F=\left[\begin{array}{cc}
0 & B s_{h} \\
\vartheta-\vartheta i_{v} & -\vartheta i_{h}
\end{array}\right]
$$

Similarly, the partial derivatives of (16) with respect to $\left(I_{h}, I_{v}\right)$ and the Jacobian matrix of $V_{i}$ at the disease - free equilibrium point (13) takes the form as

$$
V=\left[\begin{array}{cc}
\alpha+\gamma & 0 \\
0 & \delta
\end{array}\right]
$$

The inverse of the matrix $V$ is given by

$$
V^{-1}=\left[\begin{array}{cc}
\frac{1}{\alpha+\gamma} & 0 \\
0 & \frac{1}{\delta}
\end{array}\right]
$$

Now the next generation matrix $F V^{-1}$ can be constructed as

$$
F V^{-1}=\left[\begin{array}{cc}
0 & \frac{B s_{h}}{\delta} \\
\frac{\vartheta-\vartheta i_{v}}{\alpha+\gamma} & \frac{-\vartheta i_{h}}{\delta}
\end{array}\right]
$$

Hence, at the DFE point

$$
F V^{-1}\left(E_{0}\right)=\left[\begin{array}{cc}
0 & \frac{\mathrm{B}(1+\xi)}{\delta \alpha} \\
\frac{\vartheta}{\alpha+\gamma} & 0
\end{array}\right]
$$

For (21), eigenvalues are to be determined to calculate the basic reproduction number $R_{0}$ as the spectral radius of the matrix $F V^{-1}$. Thus, the eigenvalues are the solutions of the characteristic equation $\left|F V^{-1}\left(E_{0}\right)-\lambda I\right|=0$. That is,

$$
\begin{aligned}
& \left|\begin{array}{cc}
-\lambda & \frac{B(1+\xi)}{\delta \alpha} \\
\frac{\vartheta}{\alpha+\gamma} & -\lambda
\end{array}\right|=0 \\
& \Rightarrow \lambda^{2}=\left(\frac{B \vartheta}{\alpha+\gamma}\right)\left(\frac{1+\xi}{\delta \alpha}\right)
\end{aligned}
$$

$$
\Rightarrow \lambda= \pm \sqrt{\left(\frac{B \vartheta}{\alpha+\gamma}\right)\left(\frac{1+\xi}{\delta \alpha}\right)}
$$

The dominant eigenvalue of the matrix $F V^{-1}$ is

$$
\lambda=\sqrt{\left(\frac{B \vartheta}{\alpha+\gamma}\right)\left(\frac{1+\xi}{\delta \alpha}\right)}
$$

Therefore the basic reproduction number is found to have the expression

$$
R_{0}=\sqrt{\left(\frac{B \vartheta}{\alpha+\gamma}\right)\left(\frac{1+\xi}{\delta \alpha}\right)}
$$

From (22), it can be quantified that higher values of $B, \vartheta$ and $\xi$ (the immigration rate) can allow the outbreak of the disease. Conversely, for smaller values the disease dies out.

The reproduction number is a powerful parameter which measures the existence and stability of the disease in the human and mosquito populations. If $B \vartheta(1+\xi)<\delta \alpha(\alpha+\gamma)$, i.e., $R_{0}<1$, the disease-free equilibrium isthe only equilibrium and then the disease dies out. If $B \vartheta(1+\xi)>\delta \alpha(\alpha+$ $\gamma)$ i.e., $R_{0}>1$ the unique endemic equilibriumexists and the disease persists with the population.

\subsection{Stability of the Disease Free Equilibrium Point Analysis}

The equilibria are obtained by equating the right hand side of the system (10)-(12) to zero. Disease-free equilibrium (DFE) of the model is the steady-state solution of the model in the absence of the malaria disease. Hence, the DFE of the scaled malaria model (10)-(12) is given by

$$
E_{0}=\left(s_{h}^{0}, i_{h}^{0}, i_{v}^{0}\right)=\left\{\frac{1+\xi}{\alpha}, 0,0\right\}
$$

Theorem 1: The DFE, $\mathrm{E}_{0}$ of the system (10)-(12) is locally asymptotically stable if $R_{0}<1$ and unstable if $R_{0}>1$.

Proof: To find the Jacobian matrix let

$$
\begin{aligned}
f_{1}=1+\xi-\mathrm{B} s_{h} i_{v} & -\alpha s_{h}, f_{2} \\
& =\mathrm{B} s_{h} i_{v}-(\alpha+\gamma) i_{h}, f_{3}=\left[\vartheta\left(1-i_{v}\right) i_{h}\right] / \varepsilon \\
& -\delta i_{v} / \varepsilon
\end{aligned}
$$

Hence, $d s_{h} / d t=f_{1}, d i_{h} / d t=f_{2}, d i_{v} / d t=f_{3}$.

The Jacobian matrix or variational matrix is given by:

$$
J=\left(\begin{array}{lll}
\partial f_{1} / \partial s_{h} & \partial f_{1} / \partial i_{h} & \partial f_{1} / \partial i_{v} \\
\partial f_{2} / \partial s_{h} & \partial f_{2} / \partial i_{h} & \partial f_{2} / \partial i_{v} \\
\partial f_{3} / \partial s_{h} & \partial f_{3} / \partial i_{h} & \partial f_{3} / \partial i_{v}
\end{array}\right)
$$

Hence,

$$
J=\left(\begin{array}{ccc}
-\mathrm{B} i_{v}-\alpha & 0 & -\mathrm{B} s_{h} \\
\mathrm{~B} i_{v} & -\alpha-\gamma & \mathrm{B} s_{h} \\
0 & \frac{\vartheta\left(1-i_{v}\right)}{\epsilon} & \frac{-\vartheta i_{h}-\delta}{\epsilon}
\end{array}\right)
$$


Therefore the Jacobian matrix of (10)-(12) at the diseasefree equilibrium $E_{0}$ is

$$
J\left(E_{0}\right)=\left(\begin{array}{ccc}
-\alpha & 0 & -\mathrm{B}\left(\frac{1+\xi}{\alpha}\right) \\
0 & -\alpha-\gamma & \mathrm{B}\left(\frac{1+\xi}{\alpha}\right) \\
0 & \frac{\vartheta}{\epsilon} & \frac{-\delta}{\epsilon}
\end{array}\right)
$$

The characteristic equation of (23) is given by

$$
\begin{gathered}
\left|\mathrm{J}\left(\mathrm{E}_{0}\right)-\lambda I\right|=0 \\
\left|\begin{array}{ccc}
-\alpha-\lambda & 0 & -\mathrm{B}\left(\frac{1+\xi}{\alpha}\right) \\
0 & -\alpha-\gamma-\lambda & \mathrm{B}\left(\frac{1+\xi}{\alpha}\right) \\
0 & \frac{\vartheta}{\epsilon} & \frac{-\delta}{\epsilon}-\lambda
\end{array}\right|=0 \\
(-\alpha-\lambda)\left|\begin{array}{cc}
-\alpha-\gamma-\lambda & \mathrm{B}\left(\frac{1+\xi}{\alpha}\right) \\
\frac{\vartheta}{\epsilon} & \frac{-\delta}{\epsilon}-\lambda
\end{array}\right|=0 \\
(-\alpha-\lambda)\left[(-\alpha-\gamma-\lambda)\left(-\frac{\delta}{\epsilon}-\lambda\right)-\left(\frac{\vartheta}{\epsilon}\right) \mathrm{B}\left(\frac{1+\xi}{\alpha}\right)\right]=0 \\
(-\alpha-\lambda)\left[(\alpha+\gamma+\lambda)\left(\frac{\delta}{\epsilon}+\lambda\right)-\left(\frac{\vartheta}{\epsilon}\right) \mathrm{B}\left(\frac{1+\xi}{\alpha}\right)\right]=0 \\
\left(k+\left(k+\frac{\delta}{\epsilon}\right) \lambda+k \frac{\delta}{\epsilon}-k \frac{\delta}{\epsilon} R_{0}^{2}\right]=0
\end{gathered}
$$

where $k=\alpha+\gamma>0$

$$
\begin{aligned}
& (-\alpha-\lambda)\left[\lambda^{2}+\left(k+\frac{\delta}{\epsilon}\right) \lambda+k \frac{\delta}{\epsilon}\left(1-R_{0}^{2}\right)\right]=0 \\
& -\alpha-\lambda=0, \lambda^{2}+\left(k+\frac{\delta}{\epsilon}\right) \lambda+k \frac{\delta}{\epsilon}\left(1-R_{0}^{2}\right)=0
\end{aligned}
$$

Thus the eigenvalues of (2.19) are

$$
\begin{gathered}
\lambda_{1}=-\alpha ; \lambda_{2}=\frac{-\left(k+\frac{\delta}{\epsilon}\right)-\sqrt{\left(k-\frac{\delta}{\epsilon}\right)^{2}+4 k \frac{\delta}{\epsilon} \mathrm{R}_{0}{ }^{2}}}{2} ; \\
\lambda_{3}=\frac{-\left(k+\frac{\delta}{\epsilon}\right)+\sqrt{\left(k-\frac{\delta}{\epsilon}\right)^{2}+4 k \frac{\delta}{\epsilon} \mathrm{R}_{0}^{2}}}{2}
\end{gathered}
$$

It can be observed that the eigenvalues $\lambda_{1}$ and $\lambda_{2}$ are always negative quantities. However, $\lambda_{3}$ is negative if $\mathrm{R}_{0}<1$ and it is positive if $R_{0}>1$.

Recall that an equilibrium point is stable if all the eigenvalues of the next generation matrix are negatives; and unstable if at least one of the eigenvalues is positive.

Thus it can be concluded that the disease free equilibrium $E_{0}$ of the present model is stable if $\mathrm{R}_{0}<1$ and unstable if $R_{0}>1$. Hence the statement of theorem 1 is proved.

\section{Numerical Simulations}

In this section the numerical simulations of the model are carried out using a DED is cover 2.6.4and the results are presented. The initial conditions used are: $s_{h 0}=0.5, i_{h 0}=$ 0.5 , and $i_{v 0}=0.1$. The parametric values chosen for the purpose of simulation are given in Table 2 .

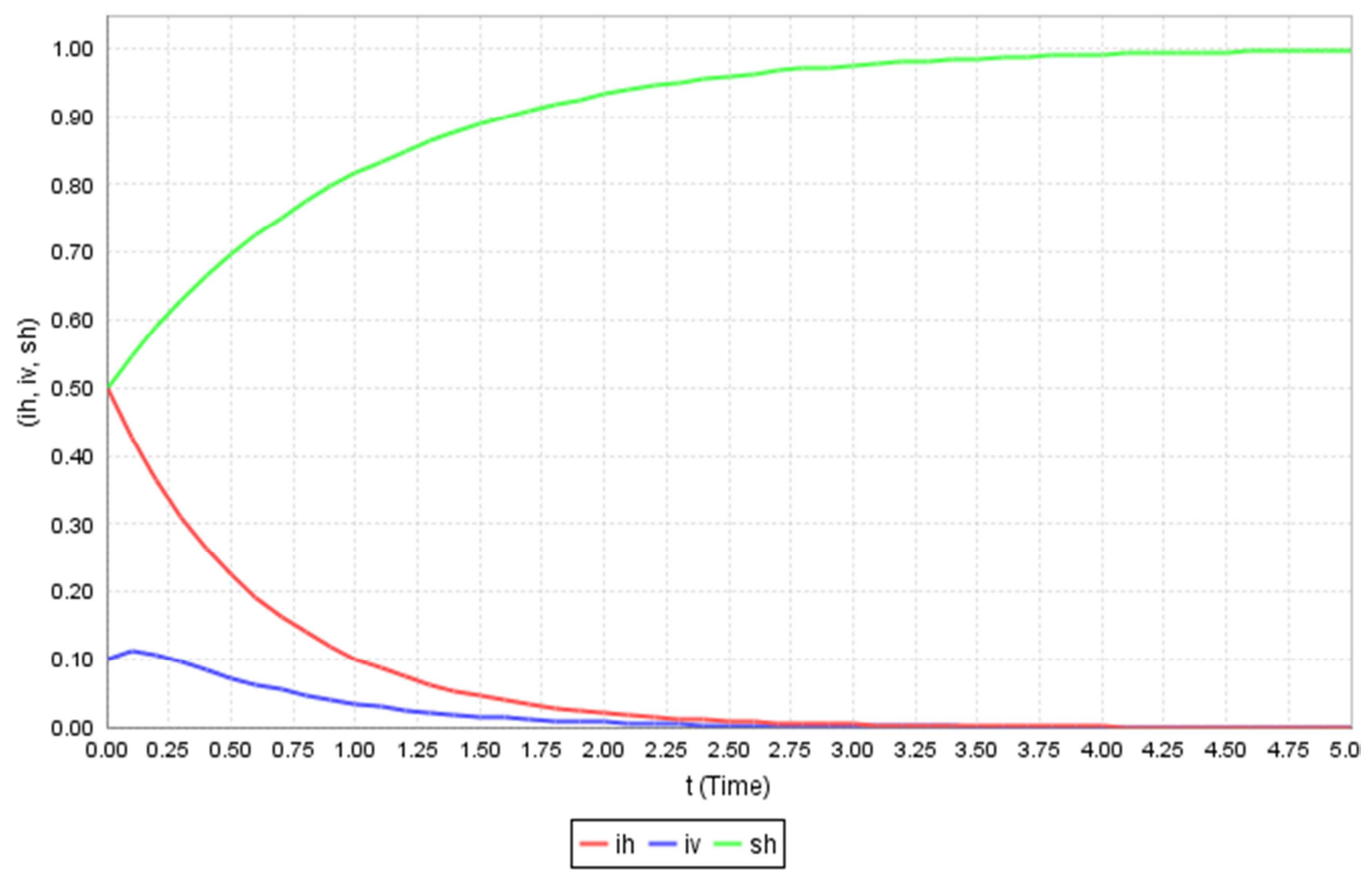

Figure 2. Numerical simulation of fraction of population with respect to time with $R_{0}=0.433013<1$. 


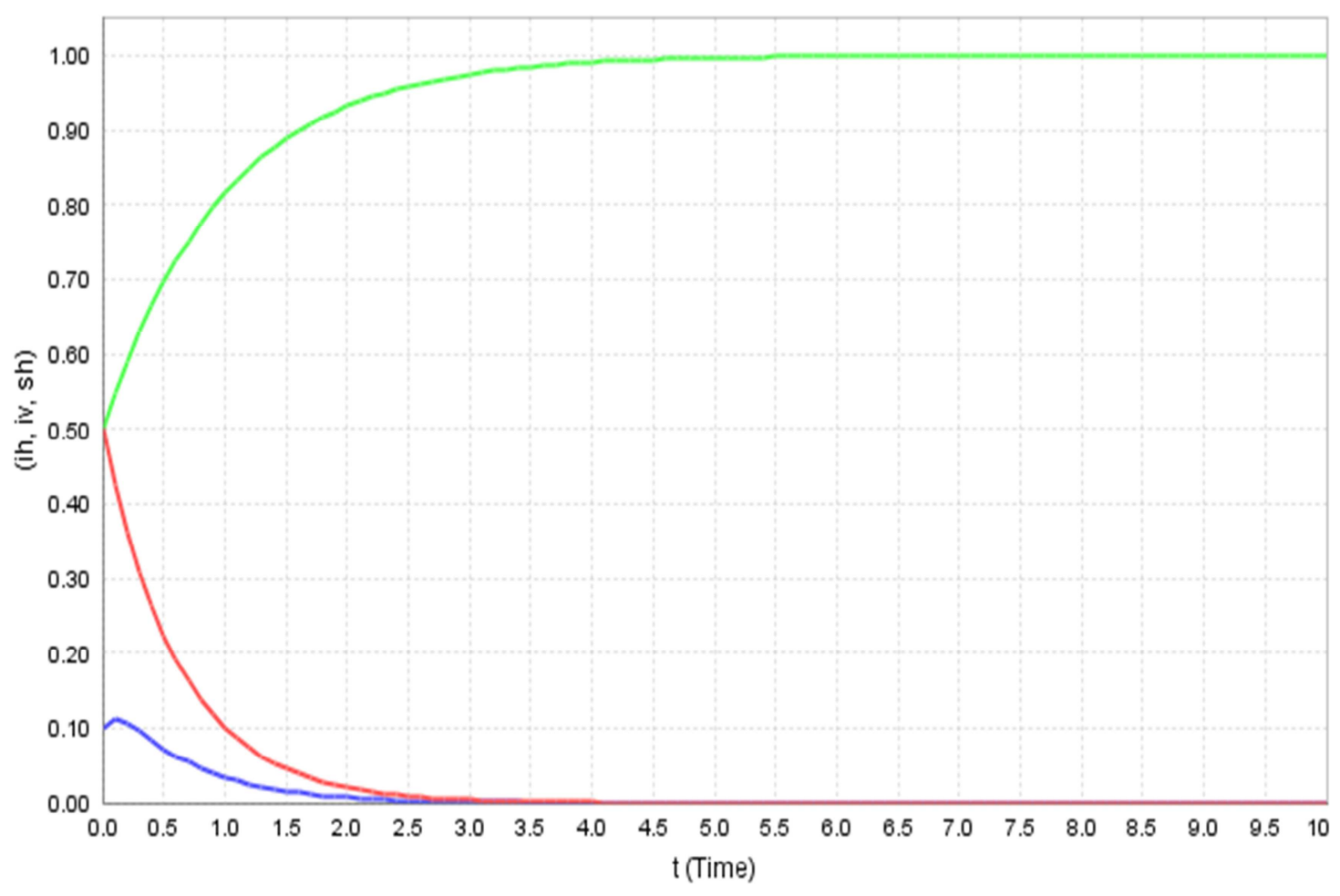

$$
\text { -ih }- \text { iv }- \text { sh }
$$

Figure 3. Numerical simulation of fraction of population with respect to time with $R_{0}=0.433229<1$.

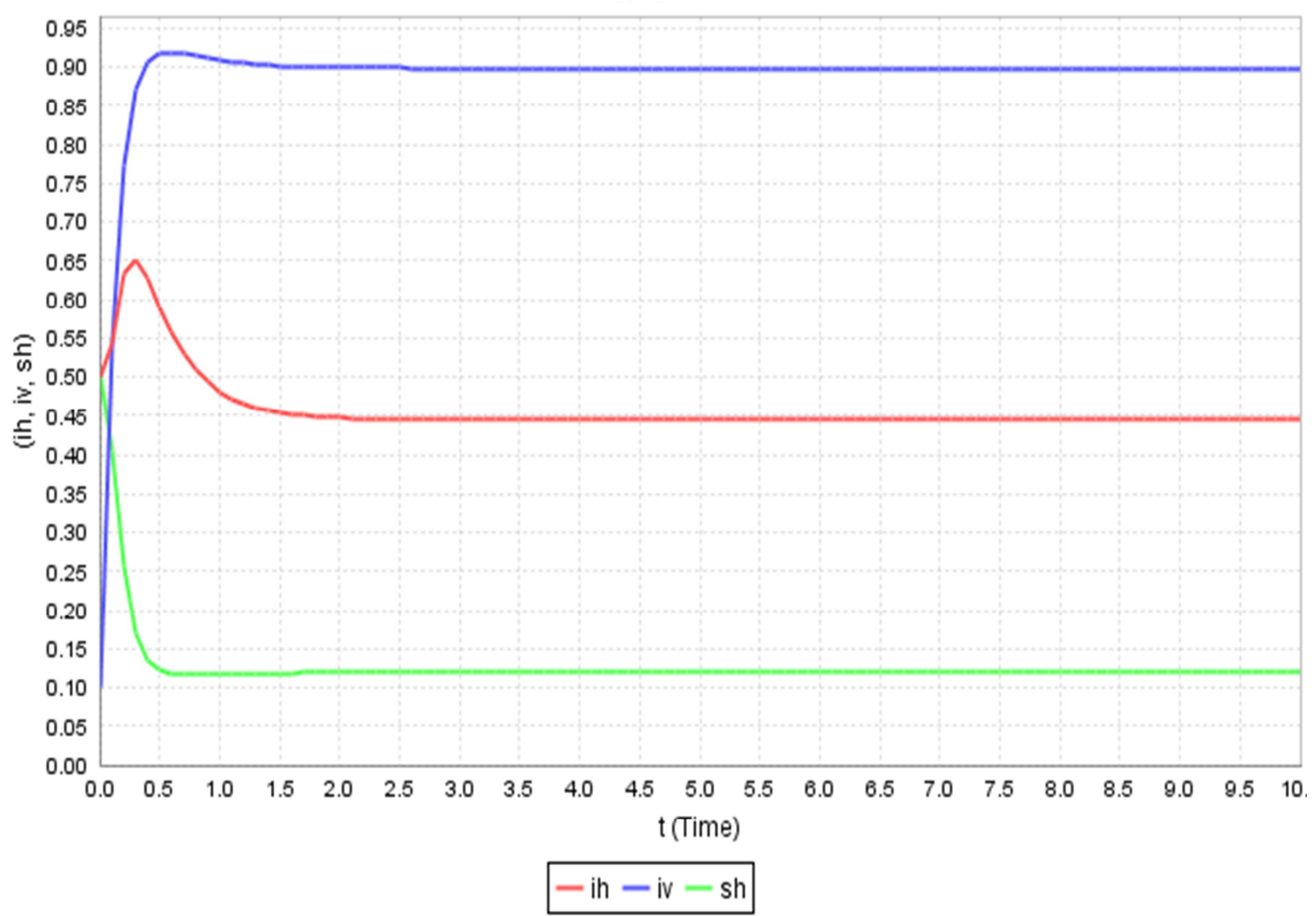

Figure 4. Numerical simulation of fraction of population with respect to time with $R_{0}=3.107277>1$. 


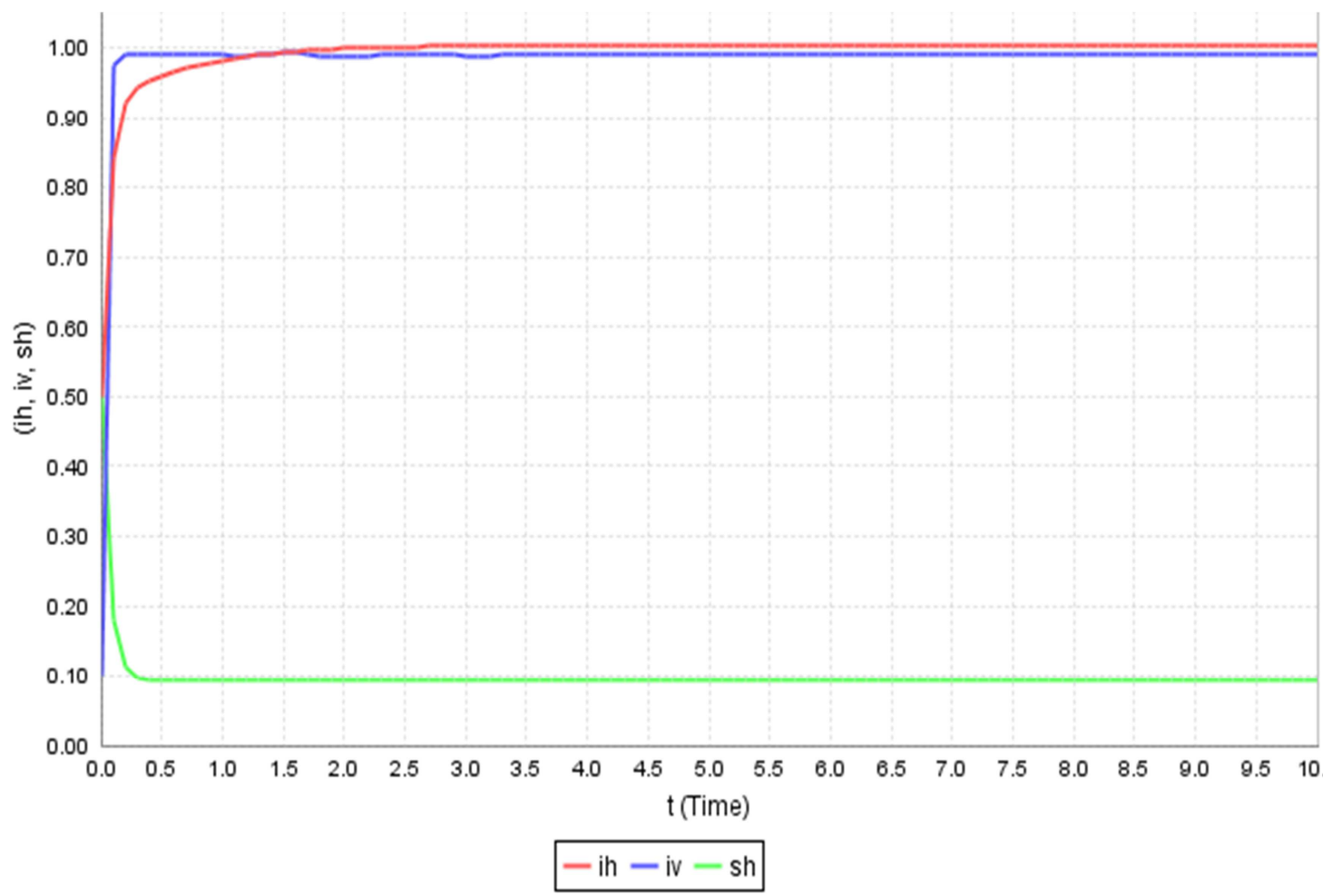

Figure 5. Numerical simulation of fraction of population with respect to time with $R_{0}=10.8562>1$.

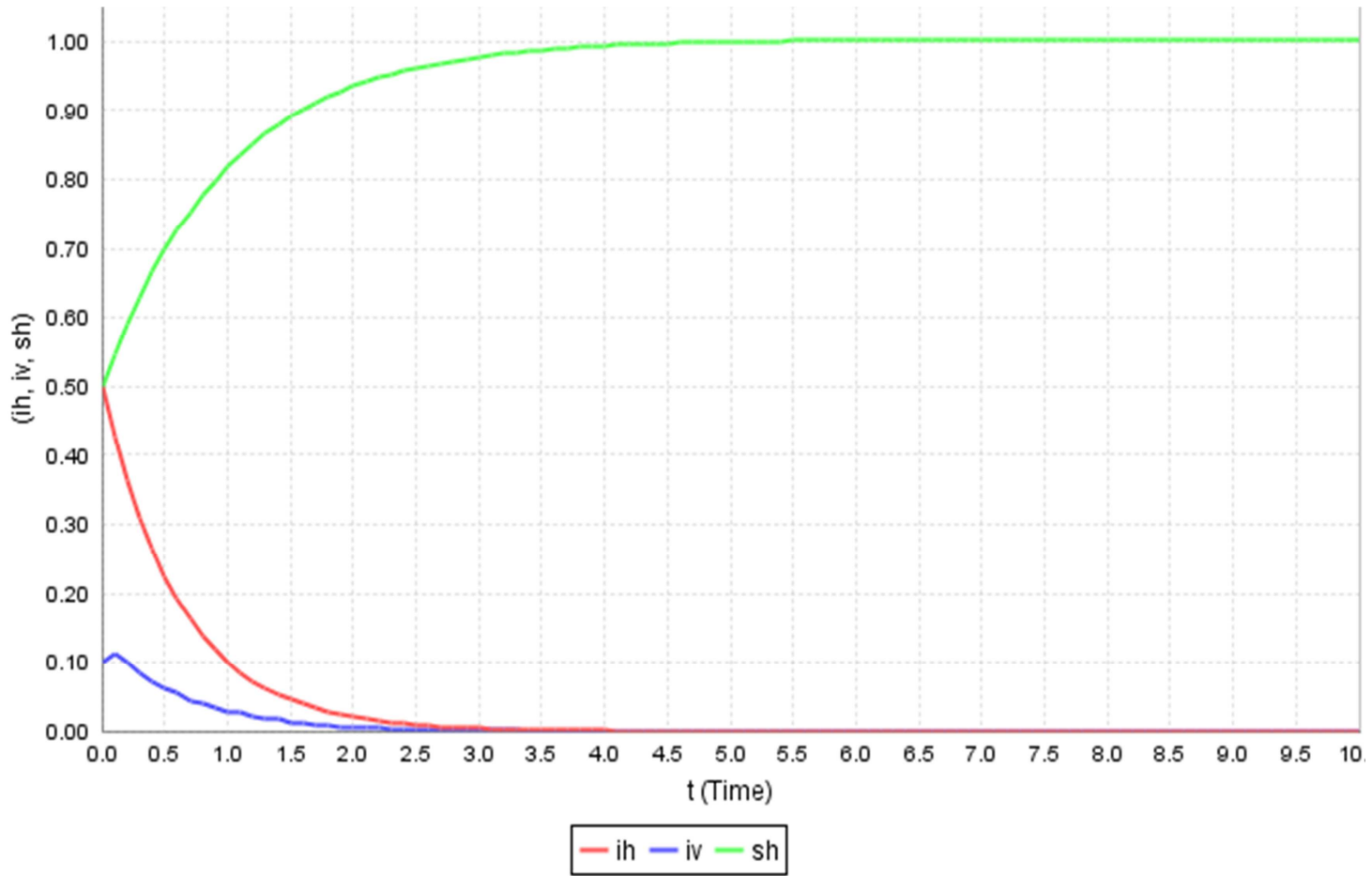

Figure 6. Numerical simulation of fraction of population with respect to time with $R_{0}=0.433662<1$.

Table 2. Parametric values used in the simulation study.

\begin{tabular}{lllllllll}
\hline Figure & B & $\boldsymbol{\alpha}$ & $\boldsymbol{\gamma}$ & $\boldsymbol{\vartheta}$ & $\boldsymbol{\delta}$ & $\boldsymbol{\epsilon}$ & $\boldsymbol{\xi}$ & $\boldsymbol{R}_{\mathbf{0}}$ \\
\hline 2 & 0.01 & 1 & 0.6 & 0.3 & 1 & 0.1 & 0 & 0.433013 \\
3 & 0.01 & 1 & 0.6 & 0.3 & 1 & 0.1 & 0.001 & 0.433229 \\
4 & 12 & 0.9 & 2 & 10 & 0.5 & 0.7 & 0.4 & 3.107277 \\
5 & 15 & 1 & 0.4 & 110 & 1 & 0.001 & 0.5 & 10.8562 \\
6 & 0.01 & 1 & 0.6 & 0.3 & 1 & 0.001 & 0.003 & 0.433662 \\
\hline
\end{tabular}




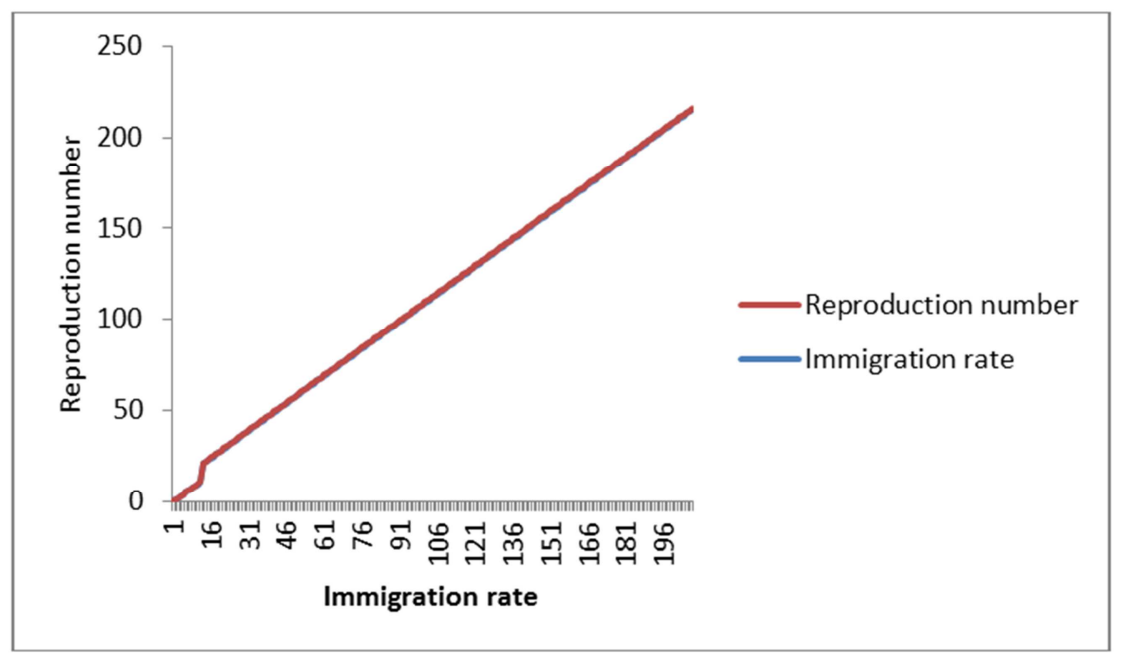

Figure 7. Reproduction number versus Immigration rate of susceptible human population.

\section{Results and Discussion}

In this paper, a SIR model is applied to malaria transmission between human and mosquito populations and studied the dynamics of the disease. The model scaled so as to reduce the number of parameters involved and to highlight the important parameters. The basic reproduction number is derived to visualize the dynamics of the disease. The existence of disease free equilibrium of model is identified and shown that it is stable.

The mathematical analysis and the simulation study shows that if the reproduction number is less than one then the DFE is locally asymptotically stable. Also in this case thought the initial sizes of human and mosquito populations are positive quantities, as the time progresses only susceptible human population remain present in the system while the other populations reduce to zero. That is, the disease dies out over a period of time. This fact is very evident in the simulation results presented in figures 2,3 , and 6 .

Further, the mathematical analysis and the simulation study also show that if the reproduction number is greater than one then the DFE is unstable. Also in this case the human and mosquito population sizes start with some positive values and as the time progresses they reach their respective carrying capacities and remain present in the system. That is, the disease does not die. This fact is very evident in the simulation results presented in figures 4 and 5.

Clearly, from the numerical simulations, the DFE is asymptotically stable whenever the reproduction number is less than one. Further, it is noticed that in order to reduce the basic reproduction number below one, it is needed to focus on reduction of the contact rates between mosquitoes and human populations, and also reduce the immigration rate.

Lastly, it is revealed that as the immigration rate increases the reproduction number increases and after certain time progress leading the reproduction number to exceed one which is shown in graph 7. Therefore the malaria disease persists in the population.

\section{Conclusion}

In this paper, a mathematical model for describing the dynamics of malaria disease is formulated taking into account the compartmentalization of both the human and mosquito populations. An SIR model with the inclusion of immigration in to susceptible compartment is formulated for human population. An SI model is formulated for mosquito population. The dynamics of mosquito population is studied along with that of the human population since the mosquito population determines whether a malaria disease will occur or not.

The transmission coefficient between susceptible humans and infected mosquitoes $\beta_{h}$; infected humans and susceptible mosquitoes $\beta_{v}$ arethe important factors in the spread of malaria.

The reproduction number being an indicator that indicates the spread of malaria is derived. By definition it is true that disease persists if the reproduction number exceeds one. The stability condition of the equilibrium points are derived and it is seen that if the reproduction number is less than one, the non-trivial equilibrium point is always locally asymptotically stable.

Simulation studies are performed by varying the values of some parameters of the model. If the immigration constant $\beta$ increases, then it will lead to an increase in the number of infected humans, giving anendemic shape to the disease. So one way to control the outbreak of malaria is to put a check on the immigration coefficient $\beta$, i.e., to curb the immigration of susceptible population.

In conclusion, there is need for effective control program against susceptible human Immigrants as they contribute to the outbreak of malaria. Spraying insecticides and proper drainage system should be a major target to eliminate mosquitoes. Early diagnosis and proper treatment with antimalarial drugs can help to control the spread of malaria. 


\section{References}

[1] Abadi Abay Gebremeskel, Harald Elias Krogstad, "Mathematical Modelling of Endemic Malaria Transmission", American Journal of Applied Mathematics, Vol. 3, No. 2, 2015, pp. 36-46. doi: 10.11648/j.ajam.20150302.12.

[2] WHO, "Investing in health research for development", Technical Report, World Health Organization, Geneva, 1996.

[3] Tumwiine J., Mugisha J., Luboobi L., "A mathematical model for the dynamics of malaria in a human host and mosquito vector with temporary immunity", Journal of Applied Mathematics and Computation, vol. 189, pp. 1953-1965, 2005.

[4] Bush A. O., Fernandez J. C., Esch G. W., Seed J. R., "Parasitism, the Diversity and Ecology of Animal Parasites", First ed., Cambridge University Press, Cambridge, 2001.

[5] Grimwade K., N. French, D. D. Mbatha, D. D. Zungu, M. Dedicoat, C. F. Gilks (2004). "HIV infection as a cofactor for severe falciparum malaria in adults living in a region of unstable malaria transmission in South Africa", Journal, vol.18, pp. 547-554.

[6] Plemmons W. R., "Mathematical study of malaria models of Ross and Ngwa", Master Thesis, University of Florida, pp. 169, 2006.

[7] Ross R., "The Prevention of Malaria”, John Murray, 1911.

[8] Macdonald G., "The Epidemiology and Control of Malaria", Oxford university press, 1957.

[9] Bailey N. T. J., "The biomathematics of malaria", Charles Gri, London, 1982.

[10] Aron J. L., "Acquired immunity dependent upon exposure in an SIRS epidemic model", Journal of Mathematical Biosciences, vol. 88, pp. 37-47, 1988.

[11] Aron J. L., "Mathematical modelling of immunity to Malaria", Journal of Mathematical Bio- sciences, vol. 90, pp. 385-396, 1988.
[12] Tumwiine J., L. S. Luboobi, J. Y. T. Mugisha, "Modelling the effect of treatment and mosquitoes control on malaria transmission", International Journal of Management and Systems, vol. 21, pp. 107-124, 2005.

[13] Yang H. M., "Malaria transmission model for different levels of acquired immunity and temperature-dependent parameters (vector)", Revista de SaudePublica, vol. 34, pp. 223-231, 2000.

[14] Ferreira M. U., H. M. Yang, "Assessing the effects of global warming and local social and economic conditions on the malaria transmission", Revista de SaudePublica, vol. 34, pp. 214-222, 2000.

[15] Koella J. C. and R. Antia, "Epidemiological models for the spread of anti-malarial resistance", Malaria Journal, vol. 2, 2003.

[16] Welch, J. Li, R. M., U. S. Nair, T. L. Sever, D. E. Irwin, C. Cordon-Rosales, N. Padilla, "Dynamic malaria models with environmental changes", in Proceedings of the Thirty-fourth southeastern symposium on system theory, Huntsville, pp. 396-400, 2014.

[17] Bacaer N. and C. Sokhna. "A reaction-diffusion system modeling the spread of resistance to an antimalarial drug", Math. Biosci. Engrg, vol. 2, pp. 227-238, 2005.

[18] Ngwa, G. A. and W. S. Shu, "A Mathematical model for endemic malaria with variable human and mosquito populations", Mathematical and Computer Modeling Journal, vol. 32, pp. 747-763, 2000.

[19] Ngwa G. A. "Modelling the dynamics of endemic malaria in growing populations", Discrete Contin. Dyn. Syst. Ser. B, vol. 4, pp. 1173-1202, 2004.

[20] Addo D. E., "Mathematical model for the control of Malaria", Master Thesis, University of Cape Coast, 2009.

[21] Yang H., Wei H., Li X., "Global stability of an epidemic model for vector borne disease", J SystSci Complex Journal, vol. 23, pp. 279-292, 2010.

[22] Miliyon Tilahun. "Backward bifurcation in SIRS malaria model”, Lsevierar Xiv: 1707.00924v3, 2017. 